Document downloaded from:

http://hdl.handle.net/10251/74251

This paper must be cited as:

Arnal Pastor, MP.; Martínez Ramos, C.; Perez Garnes, M.; Monleón Pradas, M.; Vallés Lluch, A. (2013). Electrospun adherent-antiadherent bilayered membranes based on crosslinked hyaluronic acid for advanced tissue engineering applications. Materials Science and Engineering: C. 33(7):4086-4093. doi:10.1016/j.msec.2013.05.058.

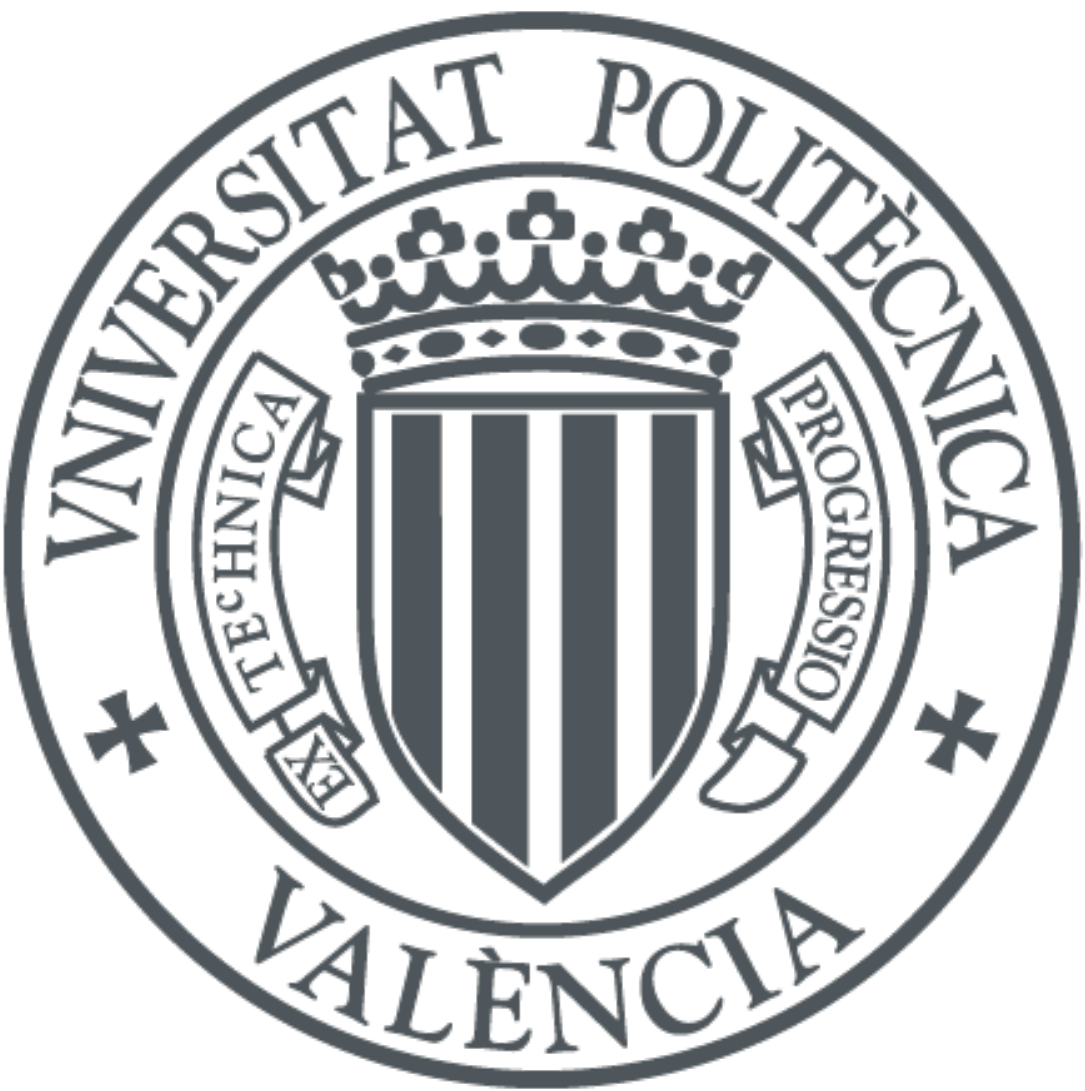

The final publication is available at

http://dx.doi.org/10.1016/j.msec.2013.05.058

Copyright Elsevier

Additional Information 


\title{
ELECTROSPUN ADHERENT-ANTIADHERENT BILAYERED MEMBRANES BASED ON CROSSLINKED HYALURONIC ACID FOR ADVANCED TISSUE ENGINEERING APPLICATIONS
}

M. Arnal Pastor ${ }^{1}$, C. Martínez $\operatorname{Ramos}^{1}$, M. Pérez Garnés ${ }^{1}$, M. Monleón Pradas ${ }^{1,2}$, A. Vallés Lluch ${ }^{1, *}$

${ }^{1}$ Center for Biomaterials and Tissue Engineering, Universitat Politècnica de Valencia, C. de Vera s/n, 46022, Valencia, Spain

${ }^{2}$ Networking Research Center on Bioengineering, Biomaterials and Nanomedicine, Valencia, Spain *Corresponding author.Tel.: +34963877277; fax: +34963877276. E-mail: mmonleon@ter.upv.es

\begin{abstract}
A procedure to obtain electrospun mats of hyaluronic acid (HA) stable in aqueous media in one single step has been developed. It consists in combining a HA solution with a divinyl sulfone one as crosslinker in a three way valve to immediately electroblow their mixture. Membranes obtained with this method, after sterilization and conditioning, are ready to use in cell culture without need of any additional post-treatment. HA nanofibers are deposited onto previously electrospun poly(Llactic acid) (PLLA) mats in order to obtain stably joined bilayered membranes with an adherent face and the opposite face non-adherent, despite their different hydrophilicity and mechanical properties. These bilayered HA/PLLA membranes may be of use, for example, in applications seeking to transplant cells on a tissue surface and keep them protected from the environment: the PLLA nanofiber face is cell-friendly and promotes cell attachment and spreading and can thus be used as a cell supply vehicle, while the HA face hinders cell adhesion and thus may prevent postsurgical adherences, a major issue in many surgeries.
\end{abstract}


Keywords: electrospinning, hyaluronic acid, crosslinking, poly(L-lactic acid), membrane

\section{Introduction}

Hyaluronic acid (HA) is one of the major components of the extracellular matrix. It is a natural linear polysaccharide composed of repeating units of $\beta-1-4-\mathrm{D}$-glucuronic acid and $\beta-1-3-\mathrm{N}$-acetylD-glucosamine [1,2]. In the last decade it has been widely proposed for many biomedical applications due to its biocompatibility, its positive influence in the healing process, the angiogenic character of its degradation products and its potential as a drug release system $[1,2,3,4,5]$.

But HA in the format of electrospun membranes has not been completely exploited yet, for two main reasons. On the one hand, HA has a poor electrospinning processability. Many different approaches have been tried in order to reduce the complexity of obtaining electrospun membranes of hyaluronic acid, among others: combining different solvents [6], assisting the process with a hot air stream [7] and blending HA with other polymers such as gelatin [8], poly(ethylene oxide) [9] or collagen $[10,11]$. On another hand, HA is soluble in water. Therefore, it is necessary to crosslink it to obtain a material stable in aqueous conditions. Many attempts have been done in this line, consisting in either a physical [12] or a chemical [13] crosslinking after the electrodeposition process, in order to obtain HA mats insoluble in water. In all these works the crosslinking was performed in a second step following the electrospinning process of the HA mat. In another approach a dual-syringe set-up was employed to combine thiolated derivatives of HA mixed with poly(ethylene oxide) (PEO) with poly(ethylene glycol) diacrylate (PEGDA) as crosslinker agent [14] during the electrospinnning process, but an additional second step was still required to remove the PEO.

In [15] divinylsulfone (DVS) was proposed as two step crosslinker for HA films, and in [16] it was successfully employed by the authors to crosslink HA coatings of acrylate scaffolds' pores in a two step process. In this work, unmodified HA has been successfully crosslinked with said crosslinker 
for the first time in one single step during the process of electrodeposition and with no additional post-treatments. To achieve this goal two solutions were combined, a HA solution and a crosslinker solution of DVS in aqueous sodium hydroxide, and the process was assisted with a hot air stream. This technique allowed obtaining very thin mats (even in the swollen state) in a single step, which are stable in phosphate buffer saline (PBS) for at least 28 days.

Still, important disadvantages of these membranes are their reduced thickness and their high equilibrium water content, which imply lack of mechanical properties and limited manageability. These drawbacks could become a problem for some tissue engineering applications. This led us to combine the HA mat with another polymer into a two-layer membrane. Here, HA has been electrospun onto previously obtained mats of poly(L-lactic acid) (PLLA), which possesses a great electrospinning processability [17], proper mechanical behavior and manipulability, and is a noncytotoxic and biodegradable FDA approved polymer [18]. Another reason to combine electrospinning of HA with PLLA is that the latter has proved to be easily colonized in vitro by different cells types, whereas HA has non-adherent properties [19] and has already been tested in clinical trials to prevent surgical adhesions [20]. The resulting HA/PLLA two-layer membrane has a hydrophilic face and a hydrophobic face, and a cell-friendly face opposite to a non-cell adhering face. Having a bilayered membrane with such differing behavior on each one of its faces could be very interesting for specific applications (Fig. 1(a)): conveying transplanted cells on the adherent face while the non-adherent face protects them from exterior aggression by macrophages; transplanting cells on a support whose other face avoids post-surgical adhesions; culturing or transplanting cells on one face (PLLA) while the HA gel on the other face continuously delivers factors or drugs of interest. Many more situations can be envisaged where the simultaneous possession of these opposing characteristics might be of advantage. However, this combination raises difficult questions to be solved, related to the hydrophilic character and mechanical properties of the two-layer system: is the interface between the independently spun PLLA and HA mats 
mechanically stable? is that interface stable once the membrane is hydrated, since both mats swell differently? how long does the HA crosslinked by this method resist dissolution in acqueous medium? These are the issues addressed in this work.

\section{Materials and methods}

\subsection{Materials}

Poly(L-lactide) (PLLA; Polylactide Resin PLA-4042D, $\mathrm{M}_{\mathrm{w}}=2,8 \cdot 10^{5} \mathrm{Da}$ ) was purchased from NatureWorks. Hyaluronic acid sodium salt (HA; 99\%, obtained from fermentation of Streptococcus equi., 1,5-1,8 $\cdot 10^{6} \mathrm{Da}$ ) was provided by Sigma. N-N dimethyl formamide (DMF; synthesis grade, Scharlau), dichloromethane (DCM; synthesis grade stabilized with $250 \mathrm{ppm}$ of BHT, Scharlau) and ethanol (EtOH; 99\%, Scharlau) were employed as solvents to prepare the electrospinning solutions. Divinyl sulfone (DVS; 97\% Sigma-Aldrich) and sodium hydroxide (NaOH; extra pure, Scharlau) were used to obtain the crosslinker solution.

\subsection{Methods}

\subsubsection{Preparation of solutions}

PLLA pellets were dissolved in a 30/70 wt\% mixture of DMF and DCM at a concentration of 5 $\mathrm{wt} \%$. After $24 \mathrm{~h}$ of stirring, PLLA pellets were completely dissolved.

HA was dissolved at a concentration of $1.87 \mathrm{wt} \%$ in a mixture of DMF, EtOH and water in the following weight proportions: $9(1 \mathrm{EtOH} / 1.5 \mathrm{DMF}) / 1 \mathrm{H}_{2} \mathrm{O}$, and stirred for $24 \mathrm{~h}$. The crosslinker solution was DVS in $0.2 \mathrm{M}$ (aq) $\mathrm{NaOH}$ at a concentration of $1.02 \mathrm{vol} \%$. 
To prepare HA films, which will be used as controls, hyaluronic acid sodium salt was dissolved at a concentration of $5 \% \mathrm{w} / \mathrm{vol}$ in sodium hydroxide $0.2 \mathrm{M}$ by shaking for $24 \mathrm{~h}$ and by stirring at 200 rpm for $2 \mathrm{~h}$.

\subsubsection{Electrospinning conditions}

A $10 \mathrm{~mL}$ syringe was loaded with PLLA solution, with a silicone tube coupling the tip and the needle. A syringe pump (Syringe pump NE-1600/NE-1800) was used to feed the polymer solution at a fixed feeding rate of $4 \mathrm{~mL} / \mathrm{h} .20 \mathrm{kV}$ were applied with a high-voltage power supply (Glassman FC Series 120 Watt). The distance between the tip of the needle and the collector was $20 \mathrm{~cm}$, the needle being in vertical position and the collector below in horizontal position. The electrodeposition time to prepare each PLLA membrane was 10 min.

Both HA and DVS solutions were placed into a $10 \mathrm{~mL}$ syringe each, with a silicone tube coupled to the tip. Two syringe pumps were used to feed the solutions at a fixed feeding rate of $5 \mathrm{~mL} / \mathrm{h}$ in the case of HA, and $1.6 \mathrm{~mL} / \mathrm{h}$ in the case of DVS. The silicone tubes coming from both solutions were connected in a three way valve in order to mix them and get the resulting solution to be electroblowed. Next, an additional tube ensured that as the mixture advanced both solutions got perfectly mixed, and allowed the crosslinking reaction to start. At the end of this tube a needle with a flat tip was inserted and tilted $40^{\circ}$ to the horizontal plane to facilitate the fiber formation, as shown

in Fig. 1(b). The collector was tilted $80^{\circ}$ with respect to the horizontal plane. The distance between the needle and the collector was $10 \mathrm{~cm}$, and the voltage applied was $27 \mathrm{kV}$. In order to facilitate the fiber formation, a stream of hot air was used. The electrodeposition time to obtain the crosslinked HA membranes varied from 60 to 90 minutes depending on their future use. The longer electrodeposition times were employed for the membranes made only of $\mathrm{HA}$ in order to ensure enough thickness to allow manipulability; the shorter times were used to fabricate the bilayered membranes. These HA/PLLA mats were obtained by electroblowing the HA-DVS solution on 
PLLA membranes previously electrospun on the collector for 10 minutes. For each layer, the conditions were the corresponding ones described above.

After electrodeposition, samples were left $24 \mathrm{~h}$ in room conditions, then dried under vacuum for 24 $\mathrm{h}$, and finally dried in vacuum and $40^{\circ} \mathrm{C}$ for $24 \mathrm{~h}$ more, to remove any residue..

\subsubsection{Preparation of hyaluronic acid discs}

The 5\% HA solution in $\mathrm{NaOH}$ was mixed with DVS at the same HA:DVS molar ratio as that of the co-electrospun materials, and stirred at $300 \mathrm{rpm}$ for $1 \mathrm{~min} .20 \mathrm{~mL}$ of the mixture were poured onto a $8.5 \mathrm{~cm}$ Petri dish. After $24 \mathrm{~h} 5 \mathrm{~mm}$-diameter discs were punched out, rinsed 5 times for $24 \mathrm{~h}$ in a 50:50 vol\% acetone: distilled water mixture and dried at $40^{\circ} \mathrm{C}$ for $5 \mathrm{~h}$ followed by $24 \mathrm{~h}$ of vacuum at room temperature.

\subsubsection{Morphological observation of the membranes with SEM}

Morphological analysis of the membranes was undertaken by scanning electron microscopy (SEM) in a JSM-6300 microscope, (JEOL Ltd., Tokyo, Japan), with the samples previously sputter-coated with gold, $15 \mathrm{kV}$ of acceleration voltage and $15 \mathrm{~mm}$ of working distance. SEM images were obtained of the surface and transversal sections. Fiber diameters were determined with the caliper accessory of the INCA suite 4.15 (Oxford Instruments plc; UK) software.

\subsubsection{Composition determination by $T G A$}

Thermogravimetric analysis was conducted with a TA-SDT Q600 device (TA Instruments, New Castle, DE, USA), from 25 to $1000^{\circ} \mathrm{C}$ at a heating rate of $10^{\circ} \mathrm{C} / \mathrm{min}$ and with a nitrogen flux of 50 $\mathrm{mL} / \mathrm{min}$, to determine the composition of the bilayer membranes in terms of mass percentage of both constituents, and their thermodecomposition profiles. Samples weighing approximately $7 \mathrm{mg}$ were dried under vacuum for $24 \mathrm{~h}$ before measurement and analyzed each time. Residual weight, 
defined as the remaining mass fraction of each sample, was recorded during its thermal degradation. Since PLLA decomposes leaving no residual weight whereas HA leaves a percentage of its initial mass as a residual weight, it was assumed for the bilayered membranes that the entire residue was a contribution from the HA component. From the percentages of residual weight for HA, $R W_{H A}$, and for the bilayered membrane, $R W_{\text {bilayered }}$, the initial mass fraction of HA in the bilayered membrane, $x_{H A}$, was quantified as $x_{H A}=R W_{\text {bilayered }} / R W_{H A}$.

\subsubsection{Contact angle measurements}

The surface wettability of both PLLA and HA sides of the membranes was characterized with $3 \mu \mathrm{l}$ extra pure water drops deposited on their surfaces in a Dataphysics OCA instrument (DataPhysics Instruments $\mathrm{GmbH}$, Filderstadt, Germany). In the case of PLLA the average contact angle of 10 water drops was calculated.

\subsubsection{Determination of dynamic and equilibrium water sorption of the membranes}

The equilibrium water sorption capacity of the membranes was evaluated by letting the samples equilibrate in a potassium iodide (KI; extra pure, Scharlau) and in a phosphate buffered saline (PBS) vapor ambient at room temperature. In the first case, the relative humidity reached was $66 \%$ [21]; in the second one it was close to 1 . Small pieces of the membranes were placed in open test tubes inside a hermetic box containing a supersaturated KI or a PBS solution, avoiding any contact of the samples with the solution. After selected times $(0,24,48,72,96,168,240,384,550,596$, 836 and 1172 hours) the samples were withdrawn and weighed in a Mettler AE 240 balance (Mettler-Toledo, Columbus, OH, USA) until no weight change was further observed. The final constant values attained were taken as the equilibrium ones. The swelling measurements were repeated with three samples each. After withdrawal, the samples were weighed and the water 
content, $W C$, calculated as $\left(m_{t}-m_{0}\right) / m_{0}$, where $m_{0}$ is the weight of the dry sample and $m_{t}$ the weight of the swollen sample after $t$ days. The equilibrium water content was defined as $E W C=\left(m_{\infty}-m_{0}\right) / m_{0}$, $m_{\infty}$ being the weight of the sample at equilibrium. Analogously, HA discs were swollen in a PBS atmosphere until equilibrium was reached. The equilibrium water content reached by these discs was used as a reference.

\subsubsection{Mechanical tensile tests}

Mats of electrospun PLLA obtained after 20 minutes of electrodeposition were cut into $0.5 \times 3 \mathrm{~cm}^{2}$ samples, their average thickness determined by SEM being $284 \pm 18 \mu \mathrm{m}$. Stretching tests were performed in the longitudinal direction on dry samples, in a stress-strain Microtest SCM3000 95 (Microtest SA, Madrid, Spain) machine at a stress rate of $0.2 \mathrm{~mm} / \mathrm{min}$ until fracture. Measurements were performed in triplicate, and the elastic modulus was determined as the initial linear slope of the strain-stress curves, the strain being obtained as the force divided by the transversal section of the specimen, and the stress as the deformation per length unit.

\subsubsection{Degradation/stability studies}

The degradation process of PLLA membranes was followed quantitatively by gel permeation chromatography (GPC), as follows. The membranes were cut into $12 \times 8 \mathrm{~mm}^{2}$ pieces, rinsed with distilled water and dried under vacuum for two days. Next, these pieces were immersed in $10 \mathrm{ml}$ of PBS each at $37^{\circ} \mathrm{C}$ for different degradation times: 0, 2, 20, 30, 50, 70, 100 and 150 days. PBS was renewed every week. After withdrawal, the degraded samples were rinsed with distilled water, dried in a vacuum desiccator and stored with silica gel until measurement.

To carry out the GPC measurements, the samples were dissolved in a $0.336 \mathrm{w} / \mathrm{v} \%$ proportion in tetrahydrofurane (THF; multisolvent GPC grade, ACS, stabilized with 250 ppm of BHT, Scharlau) by vortexing. Prior to injection the polymer solutions were clarified with the help of a nylon syringe 
filter with a $200 \mu \mathrm{m}$ pore size. The GPC analyses were performed in a Water Breeze GPC (Waters, Milford, MA, USA) equipped with Styrage HR THF columns and a Waters 2414 refractive index detector. The internal temperature of the instrument was maintained at $35^{\circ} \mathrm{C}$. The flow rate of the eluent THF was $1 \mathrm{~mL} / \mathrm{min}$, and the injection volume was $20 \mu \mathrm{L}$. The molecular weights were previously calibrated against a set of monodisperse Shodex polystyrene patterns. PLLA pellets as received were also dissolved and scanned as reference.

The stability of the HA layer and its interface with the PLLA layer was evaluated qualitatively by alcian blue-neutral red counterstaining. HA/PLLA two-layer membranes were prepared as previously described and cut into $8 \times 12 \mathrm{~mm}^{2}$ samples. These samples were conditioned with two PBS rinses of $24 \mathrm{~h}$ each, and next immersed in PBS and incubated at $37^{\circ} \mathrm{C}$ for different times: 0,1 , 2, 5, 8, 9, 12, 14, 19 and 28 days. After withdrawal, samples were stained as follows. First, samples were immersed in an alcian blue solution (at $1 \%$ in distilled water at $\mathrm{pH} 1-2,8 \mathrm{GX}$ certified, SigmaAldrich) for $10 \mathrm{~min}$, then rinsed with distilled water for $2 \mathrm{~min}$, next counter-stained with a neutral red solution $(0.3 \mathrm{~g}$ in $100 \mathrm{~mL}$ of water and $0.1 \mathrm{~mL}$ of glacial acetic acid, Scharlab) and finally rinsed with water for $30 \mathrm{~s}$. After this, PLLA remains unstained but HA acquires a brownish tone, which facilitates to identify its presence.

\subsubsection{Conditioning of the materials for cell cultures}

PLLA and HA membranes were swollen in an EtOH/ $\mathrm{H}_{2} \mathrm{O}$ 70/30 vol\% mixture before cutting them into $5 \mathrm{~mm}$ diameter discs. Then, the discs were sanitized by immersion in the $70 / 30$ vol\% $\mathrm{EtOH} / \mathrm{H}_{2} \mathrm{O}$ mixture for $3 \mathrm{~h}$. Afterwards, the $\mathrm{EtOH} / \mathrm{H}_{2} \mathrm{O}$ solution was replaced carefully by sterilized PBS with a dropper system. Several additional PBS rinses were carried out by immersion to ensure that any trace of ethanol was removed.

\subsubsection{Cell culture}


L929 cell-line of mouse fibroblasts (C34/An connective tissue, Sigma Aldrich) in its 10th passage was employed in this work. Cells reached confluence in monolayer after 3 days of culture in an F75 flask. The flask containing the cells was washed with Dulbecco's phosphate buffered saline (PBS) and incubated with $3 \mathrm{ml}$ of trypsin/EDTA (Invitrogen) for $2 \mathrm{~min}$ at $37^{\circ} \mathrm{C} .7 \mathrm{~mL}$ of Dulbecco's Modified Eagle Medium (DMEM; high glucose (4.5 g/L) Invitrogen) containing 10\% fetal bovine serum (FBS; Invitrogen) were added to neutralize the effect of the trypsin. Cells were collected and centrifuged at $1000 \mathrm{rpm}$ for $5 \mathrm{~min}$. The pellet was resuspended in DMEM and the cells were counted using a Neubauer counting chamber.

$5 \mathrm{~mm}$-diameter discs of the different mats were placed in a 96-well tissue plate. 5000 cells suspended in $20 \mu \mathrm{L}$ of medium were seeded on the upper-surface of each, and incubated for 30 min at $37^{\circ} \mathrm{C}$ and $5 \% \mathrm{CO}_{2}$. Afterwards, $180 \mu \mathrm{L}$ of fresh medium were added to each well. Samples were maintained at $37^{\circ} \mathrm{C}$, in a humidified atmosphere under $5 \% \mathrm{CO}_{2}$ for 1,7 and 14 days. The culture medium used was DMEM (4.5 g/L glucose) supplemented with 10\% FBS, $1 \%$ penicillinstreptomycin and 1\% L-glutamine, and was renewed every 2-3 days.

\subsubsection{MTS assay}

To study cell proliferation the colorimetric MTS assay ((4,5-dimethylthiazol-2-yl)-5-(3 carboxymethoxyphenyl)-2-(4-sulfophenyl)-2H-tetrazolium, Cell titer 96 Aqueous One Solution cell proliferation assay Promega, USA) was performed after 1, 7 and 14 days of incubation, three replicates per time. Briefly, at the given times samples were changed to a new 96-well plate, rinsed with PBS, and the reactant solution $(200 \mu \mathrm{L}$ containing MTS reagent/serum-free culture medium in the concentration recommended by the manufacturer) was added to each sample. Samples were incubated for $3 \mathrm{~h}$ with the reagent in the dark inside the incubator at $37^{\circ} \mathrm{C}$ and $5 \% \mathrm{CO}_{2}$. Then two $100 \mu \mathrm{L}$ aliquots from each well were pipetted into new wells. Absorbance was measured at $490 \mathrm{~nm}$ (1 s) with a Victor Multilabel Counter 1420 spectrophotometer (Perkin Elmer, Waltham, MA; 
USA). Two controls were taken in this assay, a positive (+C; MTS incubated in a polystyrene well seeded with the same amount of cells as the materials) and a negative one (MTS reagent incubated in a well without cells).

\subsubsection{Biological characterization by SEM and fluorescence microscopy}

The morphology of the L929 cells after culture was observed by SEM. After 1, 7 and 14 days of proliferation the electrospun discs were rinsed with PBS and then fixed with 4 vol\% glutaraldehyde (Scharlab) for $30 \mathrm{~min}$ at $4^{\circ} \mathrm{C}$. After fixation samples were rinsed twice with PBS. A post-fixation treatment was applied: samples were incubated with 2 vol\% osmium tetraoxide for $2 \mathrm{~h}$ at room temperature following four rinses with distilled water. After post-fixation, samples were dehydrated through a series of graded ethanol $(30,50,70,96$, and 100$)$ at $4^{\circ} \mathrm{C}$. Samples were dried by criticalpoint drying. After sputter-coating samples with gold, they were examined in a Hitachi S-4800 SEM device (Hitachi High-Technologies Canada Inc., Toronto, Canada) at $20 \mathrm{kV}$ and $15 \mathrm{~mm}$ of working distance.

Cells proliferation and distribution was also studied by fluorescence microscopy. After culture, the samples were rinsed with PBS, fixed with 4 vol\% formalin for $1 \mathrm{~h}$ at $4^{\circ} \mathrm{C}$, and washed twice with PBS for 5 min. Cells were permeabilized with 0.5 vol\% Triton X-100 for 5 min at room temperature. After two rinses with Dulbecco's Phosphate-Buffered Saline (DPBS), the samples were incubated with BIODIPY-FL phallacidin (Invitrogen) for $1 \mathrm{~h}$ at room temperature in the dark, next washed twice with DPBS and counterstained for 5 min with 4',6-diamidino-2-phenylindole dihydrochloride (DAPI; Sigma, 1/5000). Finally, the discs were rinsed twice with PBS and mounted with Fluorsave reagent (Calbiochem) coverslipped, and stored in the dark at $4^{\circ} \mathrm{C}$ until microscopy observation in a Leica DM6000 inverted microscope.

\subsubsection{Statistical analysis}


The results from all the experiments are expressed as mean \pm standard deviation. Data were analyzed pair wise with ANOVA test with Statgraphics Centurion XVI.I. Results are statistically significant unless noted otherwise. Significance was assigned at $\mathrm{p}$-values $<0,05$.

\section{Results and discussion}

\subsection{Morphological, physico-chemical and mechanical characterization}

Electrospun mats of PLLA obtained with the conditions previously described had a very low density of defects and a uniform random fiber distribution, as Fig. 2(a) shows. The mean diameter of the fibers was $751 \pm 211 \mathrm{~nm}$, measured on at least 100 fibers with the INCA suite 4.15 software. A histogram plotting the fibers diameter distribution is shown in Fig. 3(a). The fiber diameters are concentrated in two main intervals, above $900 \mathrm{~nm}$ and between 600 and $700 \mathrm{~nm}$.

[Figures 2 and 3]

In obtaining electrospinning of HA two factors were found to be essential: to assist the process with warm air blowing, and to tilt the needle. The warm air helps the formation of the fibers as reduces the viscosity, contributes to solvent evaporation and shear-stretches the drop of the polymer solution facilitating the formation of the jet. The disadvantage of using this air blow is that it introduces instabilities in the process of electrodeposition.

The fibers obtained in the case of the electrospun HA are smaller, $202 \pm 48 \mathrm{~nm}$ in diameter (measured on at least 40 fibers), Fig. 2(c) and 3(b). In this case the density of defects was greater, because of instabilities during the process, but the distribution of the fiber diameters is more uniform and narrow, being 150-200 nm the preferred interval.

The critical issues of the electrospun HA membranes are the small thickness obtained and the difficult manipulation of the membranes in the swollen state. In order to overcome them and at the same time obtain a bifunctional two-layer membrane, the antiadherent HA mat was combined with 
an electrospun mat of PLLA, Fig. 2(b), where the PLLA layer is suitable for seeding cells. The elastic modulus of the PLLA membranes under traction was found to be $17.8 \pm 1.2 \mathrm{MPa}$, high enough to provide the necessary mechanical strength and facilitate manipulability of the bilayered membranes for their implantation [22]. The average thickness of the bilayer membranes obtained here after an electrodeposition time of 10 min for PLLA and 60 min for HA was $235 \pm 40 \mu \mathrm{m}$, but this thickness could be tailored changing the electrospinning times (thicker membranes could be obtained by increasing the electrodeposition time [23]).

The composition of the bilayered membranes was determined with the help of the thermal decomposition thermograms. The residual weight $(R W)$ is plotted against temperature in Fig. 4, showing that PLLA decomposes in a temperature window between 280 and $380^{\circ} \mathrm{C}$ leaving no solid residue, whereas HA decomposes earlier and in three steps: after a smooth weight decrease between 50 and $200^{\circ} \mathrm{C}$ a marked drop of a $30 \%$ of weight occurs, followed again by a slight decrease of weight from 275 to $500^{\circ} \mathrm{C}$, leaving a considerable solid residue of $34.0 \pm 1.7 \%$ of weight. The bilayered membrane decomposes at intermediate temperatures, with an initial slight weight loss, the main drop of $80 \%$ of weight from 200 to $320^{\circ} \mathrm{C}$, and another slight loss until $450^{\circ} \mathrm{C}$, leaving a solid residue weighing $7.4 \pm 0.6 \%$ of the initial mass, approximately. This residue, attributable in its entirety to the HA in the membrane, allows the determination of the initial mass fraction of HA in the sample, as explained previously. The HA mass fraction in bilayered membranes obtained by 10 min of electrodeposition of PLLA and $60 \mathrm{~min}$ of HA is $21.8 \%$. If required, this proportion could be modulated by changing one or both electrodeposition times, and easily determined by this technique.

[Figure 4]

Electrospun PLLA membranes have a water contact angle of $130,4 \pm 2,5^{\circ}$, typical of a scarcely wettable material. On the contrary, the HA extraordinary wettability led to a very fast absorption of the water drops and even to the folding of the vicinities, which impeded accurate contact angle 
measurements. Nonetheless, photographs of the water drops over the HA and PLLA mats have been included as insets in Fig. 2 to illustrate their different shape.

Both layers of the membrane remained joined even when swollen by immersion in PBS, despite the different hydrophilicity of their components. This remarkable stability of the interface between both layers may be explained by the difference of typical fiber diameters of both components, which would allow the HA nanofibers to entangle around previously electrospun PLLA nanofibers as they are electrodeposited, defining in this way a stably joined interface between both layers.

The water uptake of the electrospun HA (PLLA does not swell in aqueous media) samples was determined in two atmospheres of different water activity (over saturated salt solutions of PBS and of KI, respectively) for different times up to 50 days. These results are shown in Fig.5. The swelling in the PBS and the KI atmospheres is markedly different $(p<0,05)$ because of the different water activities. In the case of PBS atmosphere an equilibrium water content of approximately 5.3 was achieved after 25 days whereas in the KI atmosphere, as expected given the lower water activity, the $E W C$ is much lower and around 0.3. These results have been compared with those obtained with HA films crosslinked in a mold and cast. In this case an $E W C$ of $2.86 \pm 0.28$ was obtained in the PBS atmosphere, lower than that achieved with electrospun crosslinked HA. This indicates that the crosslinking degree is lower under the electrospinning conditions, due to the much shorter time available for both co-electrospun solutions to be in contact and thus for the crosslinking reaction to take place. On the contrary, during the preparation of HA films the DVS is able to diffuse efficiently throughout the HA solution and crosslink the HA molecules, for the evaporation of water is in this case much slower.

The data from the kinetics of swelling in PBS atmosphere were adjusted to Fick's diffusion equation. This equation predicts a linear dependence of the normalized water uptake at time $t,\left(m_{t^{-}}\right.$ $\left.m_{0}\right) /\left(m_{\infty}-m_{0}\right)$, on the square root of time over the thickness of the slab through which the mobile species diffuses, $t^{0.5} / \ell$ [24]. In the case of our electrospun mats such a slab is not a bulk one, but 
consists of nanofibers leaving empty spaces between them; its thickness, as measured with a micrometer, is thus an 'apparent' one. This apparent thickness was employed in Fick's equation in order to estimate $D$ (see inset of Fig. 5). With this proviso the water absorption followed closely a Fickian behavior, with $D=4.72 \cdot 10^{-9} \mathrm{~mm}^{2} / \mathrm{s}$.

\section{[Figure 5]}

To assess the efficiency of the crosslinking of HA during the electrodeposition process, a test of immersion of the bilayered membranes in PBS was carried out for different times up to 28 days. As previously described, after each selected time an alcian blue-neutral red counter-staining (as specific stain for mucopolysaccharides) was performed. This staining clearly revealed that after 28 days the HA layer had not dissolved or hydrolysed, Fig. 6. This result proved that HA had been successfully crosslinked during the co-electrospinning process, and that in the HA/PLLA two-layer membranes both layers remained bonded for more than the time needed for most potential applications.

\section{[Figure 6]}

Since PLLA is meant to provide the shape and integrity of the bilayered membranes, particularly when swollen, its degradation pattern is a crucial aspect to be investigated, especially taking into account the large specific area of the mats exposed to the aqueous medium. Electrospun PLLA membranes were thus degraded for different times up to 150 days and the weight-average molecular weight $\left(M_{w}\right)$ was determined for each by GPC (Fig. 7). From 100 days on, membranes became more fragile and brittle on handling. Contrary to what might have been expected for this fibrous structure, the molecular weight did not decrease sharply but linearly from the beginning up to $70 \%$ after 150 days. Similar results were obtained in [25] for PLLA scaffolds having a porosity around 94\%, in which $M_{w}$ dropped around $50 \%$ in 40 weeks

[Figure 7] 


\subsection{Biological experiments}

The MTS assay was carried out after 1, 7 and 14 days of cell culture on PLLA and HA membranes as an indicator of the metabolic activity (Fig. 8). The results show that none of the materials used was cytotoxic. In the electrospun PLLA cells proliferate much during the first week and less, if at all, in the second week, likely because they reach confluency. The trend was similar in the positive control, though with more metabolic activity for a larger amount of surviving cells on seeding. On the contrary, the results obtained for HA show a very poor initial adhesion (as day 1 MTS results indicate), and a slight proliferation after 7 or 14 days, but far from the levels reached in PLLA or the positive control. This later increment of cell viability on the HA mats could be attributed to the delayed cumulative effect of ECM protein deposition by the surviving cells onto the material, which would gradually improve its initial very low adherence ability for cells.

[Figure 8]

SEM images (Fig. 9) confirmed what was hypothesized after the MTS results. Initial adhesion was better in electrospun PLLA whereas very few cells were attached to the HA surface, as expected. After 7 or 14 days, the PLLA surface was completely covered; cells reached confluence in a spread conformation. By contrast, on the HA cells proliferated in aggregates with a more rounded conformation; apparently cells preferred to stick onto each other, eventually shaping spheres, rather than colonizing and spreading on the HA surface. Another aspect to be highlighted is that coelectrospun HA maintained its nanofibrillar structure even after 14 days of cell culture and the treatments involving sample preparation for SEM, Fig. 9(h).

\section{[Figure 9]}

DAPI and phallacidin staining of the cells, Fig. 10, lend further support to the conclusions suggested by the SEM results, Fig. 9. On PLLA mats the cells proliferated up to confluence in a uniform cell layer, whereas on HA mats initial cell adhesion was very poor and the surviving cells proliferated but forming rounded aggregates. These results confirm that PLLA membranes have 
suitable biological properties, and electrospun HA could be incorporated as an effective antiadherent external layer.

[Figure 10]

The results obtained suggest that these bilayered membranes could find a variety of applications in which different behaviors in each face in terms of hydrophilicity, mechanical strength, biodegradation rate or cell attachment are sought in a single construct. As for HA, in combination with carboxymethylcellulose it has been successfully tested in preclinical trials to prevent cardiac adhesions [26], demonstrating that HA membranes might have a potential application as preventive of post-surgical adhesions. Taken together with previous works in which PLLA combined with other materials has been investigated for cardiac tissue engineering purposes [27], this leads us to think that the PLLA/HA combination could develop suitably as a cardiac patch, where the PLLA internal (myocardial) face worked as cell supplier and the HA external one to reduce post-surgical adhesions.

\section{Conclusions}

The co-electroblowing procedure herein developed allows the fabrication of non-soluble HA nanofiber mats in a single step. It consists in mixing a HA solution with another of the crosslinker in a three-way valve just before electrospinning. These nanofibers can be electroblowed stably on previously electrospun tougher PLLA mats to obtain bilayered membranes. The flexibility of the fabrication process allows the preparation of membranes with different thicknesses and properties by varying the electrospinning times.

The HA crosslinking efficiency on electrospinning was found to be lower than that achieved when films are obtained by solvent casting keeping the same DVS: HA ratio, probably due to a much shorter reaction time; nevertheless, the degree of crosslinking of the nanofibers was enough to 
obtain membranes that were stable in PBS for at least 28 days and remained assembled with the PLLA layer. As regards their biological performance, these membranes are non-cytotoxic; the PLLA mats promote cell adhesion, proliferation and spreading on their fibers, whereas the HA ones inhibit cell adhesion and proliferation occurs in rounded aggregates poorly attached.

\section{Acknowledgements}

The authors acknowledge the financing through project FP7 NMP3-SL-2009-229239 "Regeneration of cardiac tissue assisted by bioactive implants" (RECATABI). 


\section{Figure captions}

Figure 1. a) Schematic representation of the obtained bilayer membrane, and b) sketch of the employed electrospinning setup.

a)

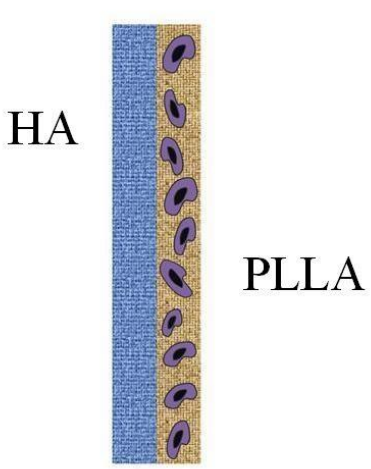

b)

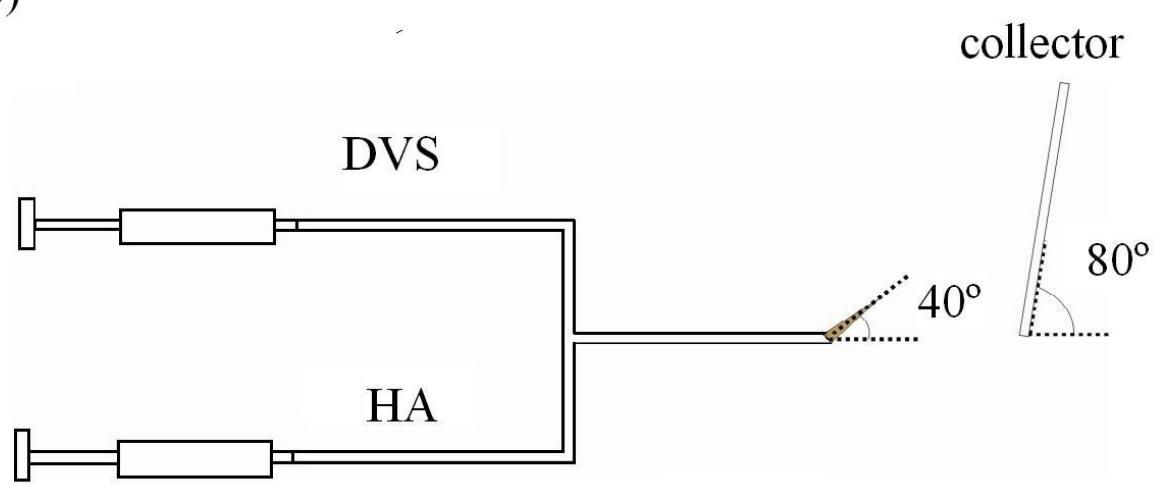

Figure 2. SEM micrographies of the electrospun bilayer membranes: (a) PLLA surface, (b) transversal section (up: HA layer, down: PLLA layer) and (c) HA crosslinked surface. Insets in (a) and (c) show $3 \mu \mathrm{l}$ water drops on the electrospun PLLA and crosslinked HA surfaces, respectively.
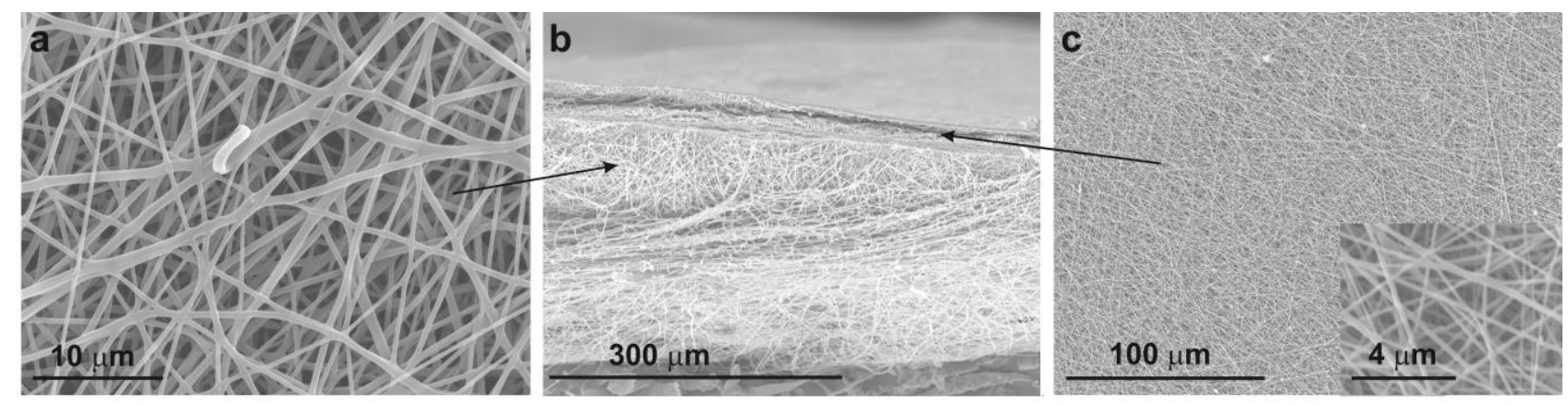

Figure 3. Histograms of the (a) PLLA and (b) crosslinked HA fiber diameters. 

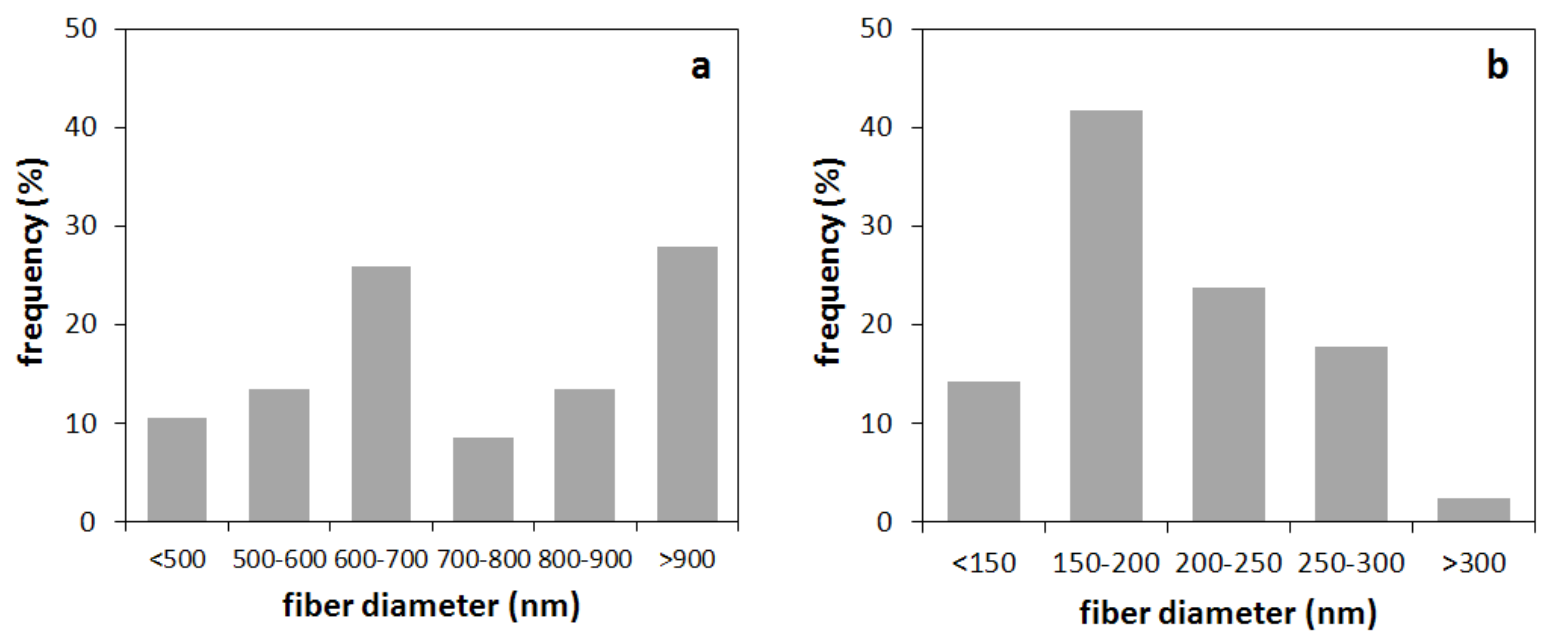

Figure 4. Thermogravimetric curves of electrospun PLLA membranes, co-electrospun HA-DVS membranes and bilayered membranes, showing the residual mass fraction as a function of the temperature.

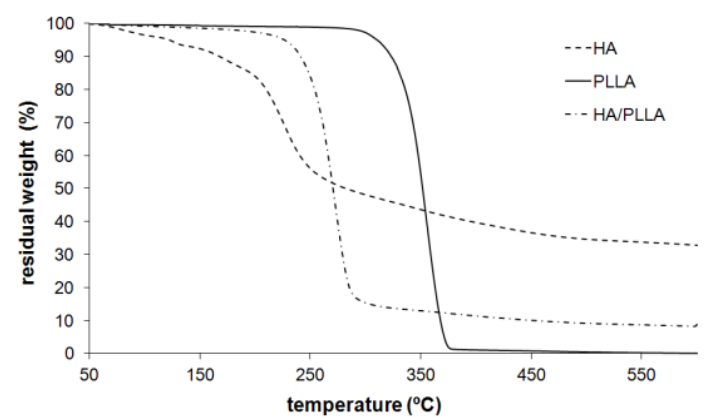

Figure 5. Water content of co-electrospun HA/DVS mats as a function of time in PBS and KI atmospheres. Inset: Plot used for comparison with the Fick equation.

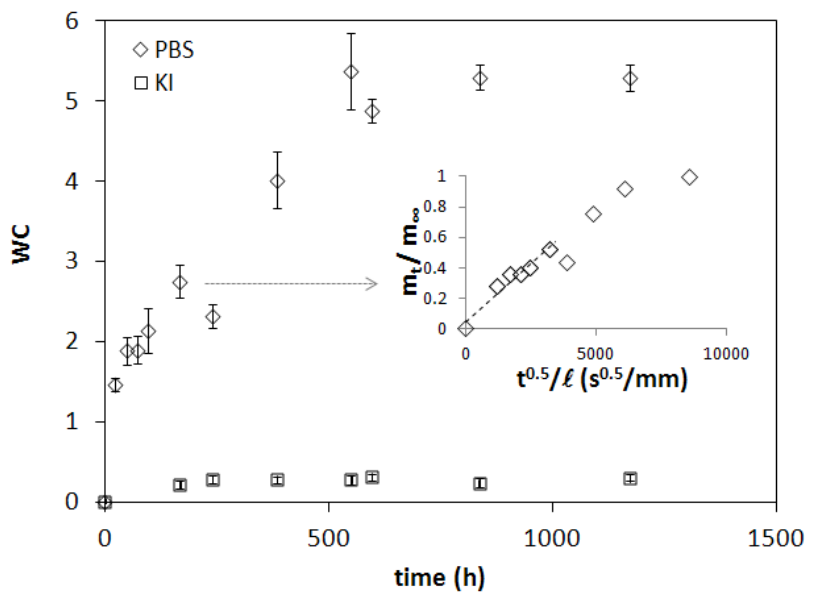


Figure 6. Photographs of bilayered membranes counterstained with alcian blue and neutral red taken from the HA-DVS side, after immersion in PBS at $37^{\circ} \mathrm{C}$ for: (a) 0, (b) 5, (c) 12, (d) 19 and (e) 28 days; (f) taken from the PLLA side as control. Unstained white color stands for PLLA and brown for HA.

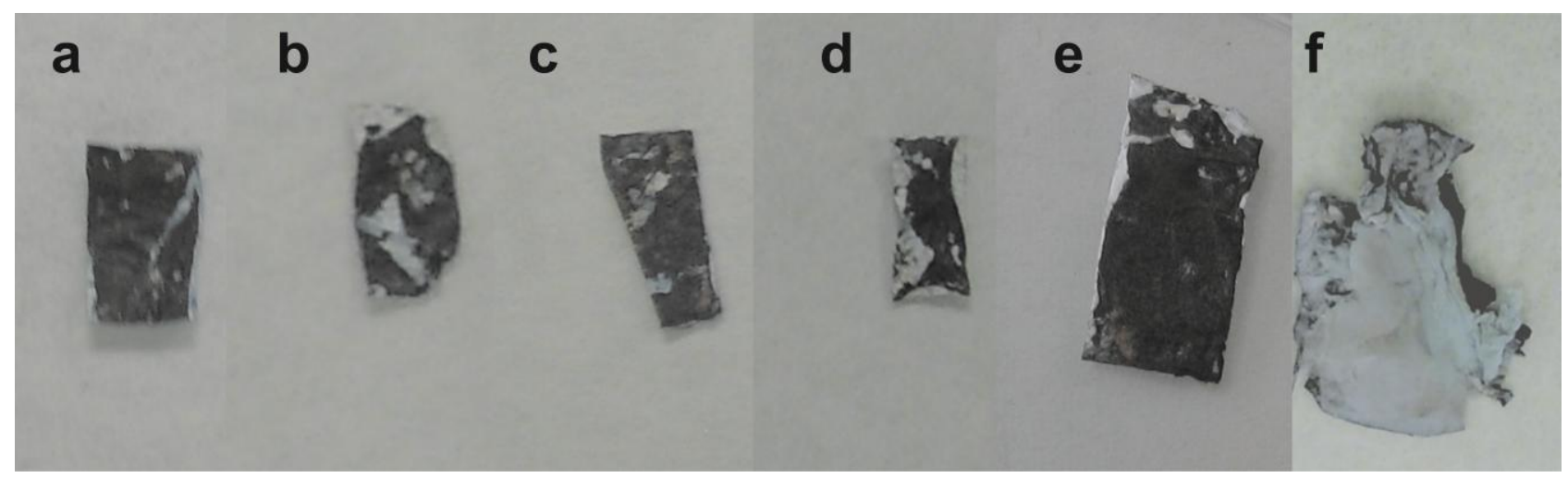

Figure 7. Dependence of the weight-average molecular weight of electrospun PLLA with the degradation time in $\mathrm{PBS}$ at $37^{\circ} \mathrm{C}$.

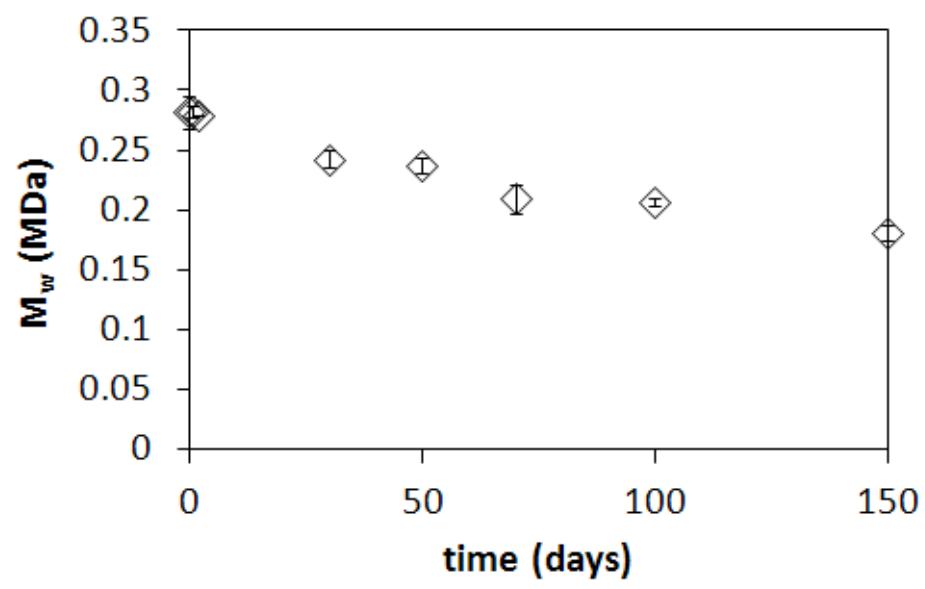

Figure 8. MTS results of L929 cells on electrospun PLLA mats, co-electrospun HA-DVS mats, and a well plate as positive control $(+\mathrm{C})$ after 1,7 and 14 days of culture. \# corresponds to nonsignificant differences $(\mathrm{p}>0,05)$. 


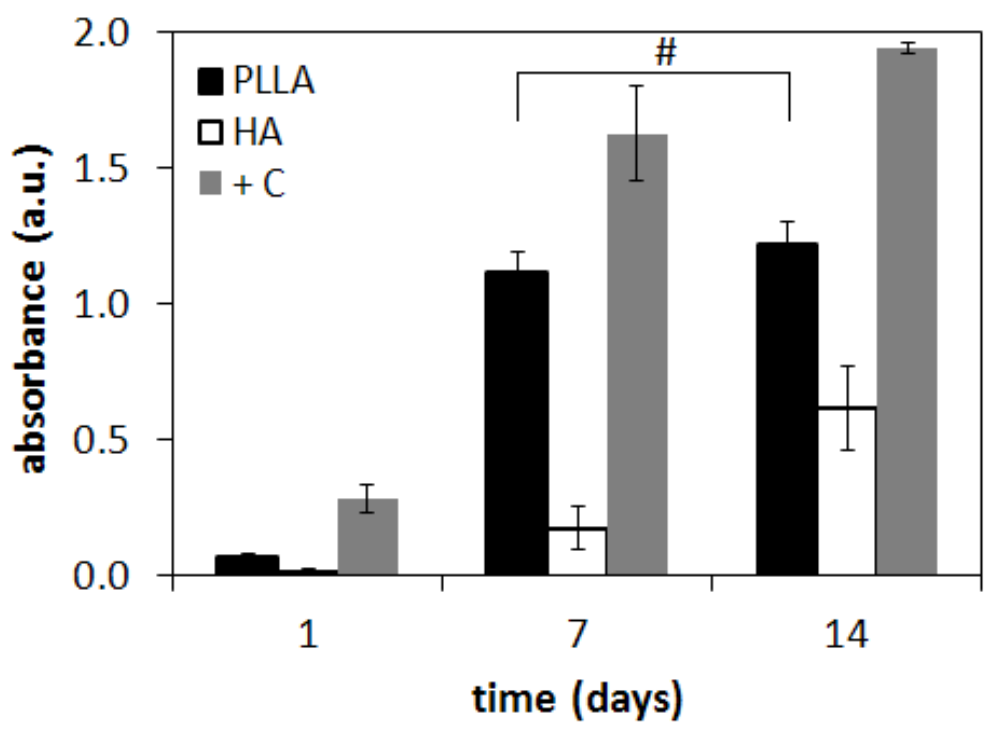

Figure 9. SEM images of L929 cells cultured on (a-d) electrospun PLLA mats and (e-h) coelectrospun HA/DVS mats for: (a, e) 1, (b, f) 7 and (c, d, g, h) 14 days.
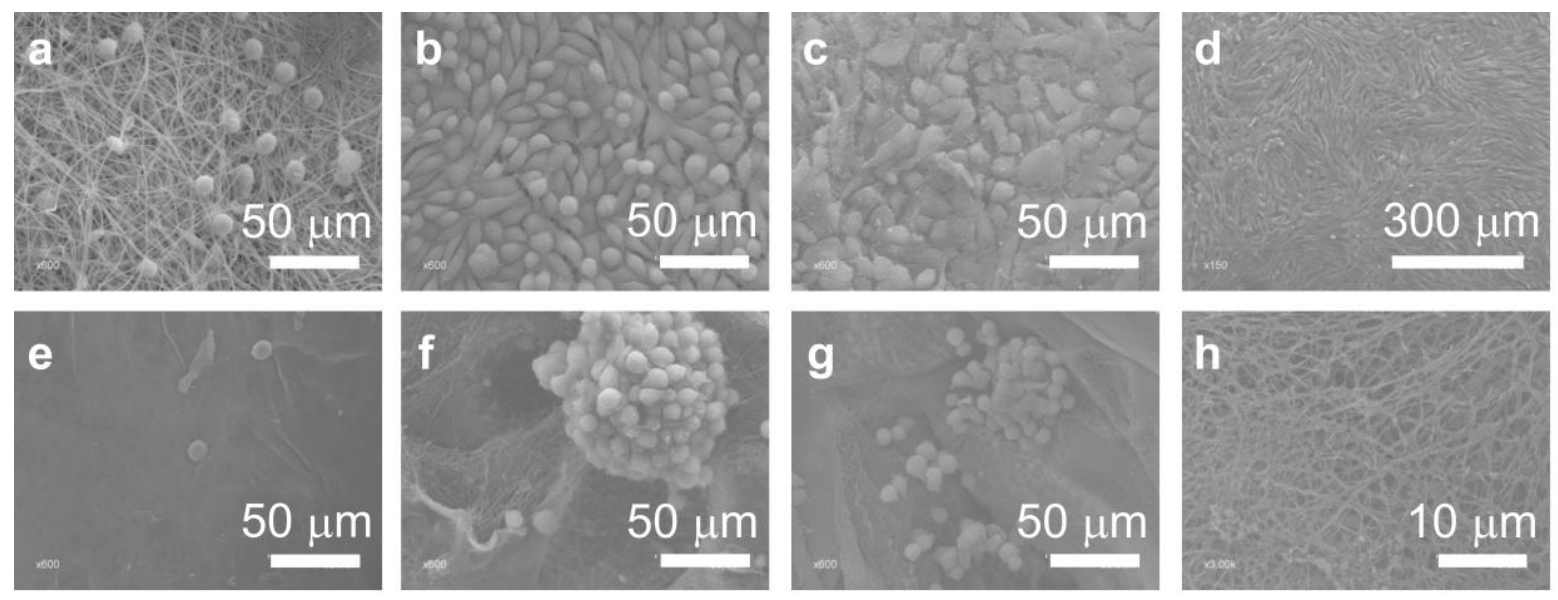

Figure 10. Fluorescence microscopy of immunostained cultures showing the distribution of the L929 cells on (a-c) electrospun PLLA mats and (d-f) co-electrospun HA/DVS mats after (a, d) 1, (b, e) 7 and (c, f) 14 days of culture, after DAPI (blue) and phallacidin staining (green) for nuclei and actin, respectively. 


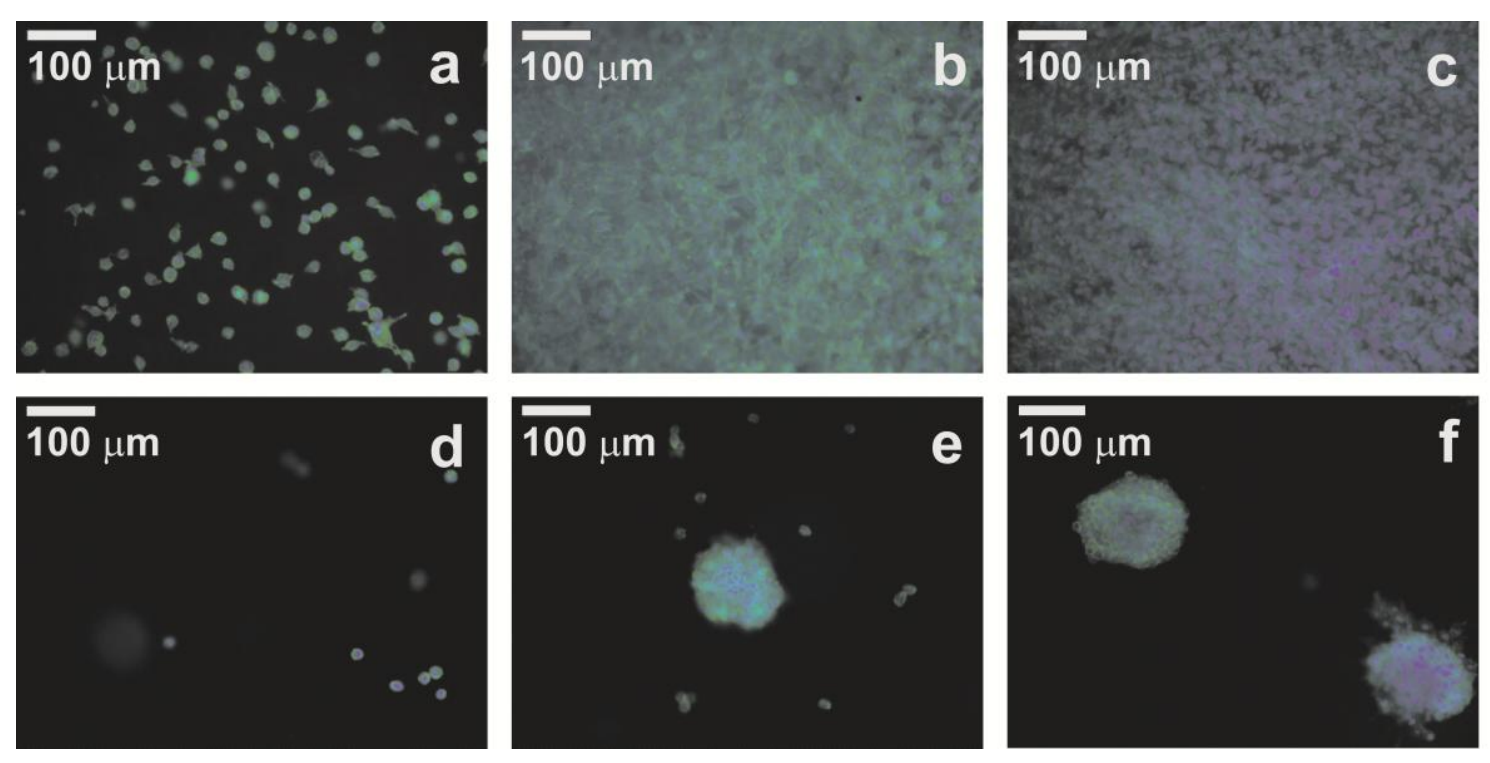




\section{References}

[1] Brenner EK, Schiffman JD, Thompson EA, Toth LJ, Schauer CL. Electrospinning of hyaluronic acid nanofibers from aqueous ammonium solutions. Carbohyd Polym 2012; 87: 926-9.

[2] Garg HG, Charles A, Hales CA. Chemistry and biology of hyaluronan. Elsevier, 2004.

[3] West DC, Hampson IN, Arnold F, Kumar S. Angiogenesis induced by degradation productss of hyaluronic acid. Science 1985; 228: 1324-6.

[4] Gaffney J, Matou-Nasri S, Grau-Olivares M, Slevin M. Therapeutic applications of hyaluronan. Mol. BioSyst. 2010; 6: 437-43.

[5] Liu Y, Ma G, Fang D, Xu J, Zhang H, Nie J. Effects of solution properties and electric field on the electrospinning of hyaluronic acid. Carbohyd Polym 2011; 83: 1011-15.

[6] Li J, He A, Han CC, Fang D, Hsiao BS, Chu B. Electrospinning of hyaluronic acid (HA) and HA/gelatin blends. Macromol. Rapid Comm 2006; 27: 114-20.

[7] Um IM, Fang, D, Hsiao BS, Okamoto A, Chu B. Electro-spinning and electro-blowing of hyaluronic acid. Biomacromolecules 2004; 5: 1428-36.

[8] Li J, He A, Zheng J, Han CC. Gelatin and Gelatin-Hyaluronic acid nanofibrous membranes produced by electrospinning of their aqueous solutions. Biomacromolecules 2006; 7: 2243-2247.

[9] Jia Y, Ghoshb K, Shuc XZ, Lia B, Sokolova JC, Prestwichc GD, Clarkb RAF, Rafailovich MH. Electrospun three-dimensional hyaluronic acid nanofibrous scaffold. Biomaterials 2006; 27: 378292.

[10] Hsu FY, Yi-Sheng Hung YS, Liou HM, Shen CH. Electrospun hyaluronate-collagen nanofibrous matrix and the effects of varying the concentration of hyaluronate on the characteristics of foreskin fibroblast cells. Acta Biomater 2010; 6: 2140-7. 
[11] Kim TG, Chung HJ, Park TG. Macroporous and nanofibrous hyaluronic acid/collagen hybrid scaffold fabricated by concurrent electrospinning and deposition/leaching of salt particles. Acta Biomater 2008; 4: 1611-9.

[12] Wang X, Um IC, Fang D, Okamotoc A, Hsiao BS, Chu B. Formation of water resistant hyaluronic acid nanofibers by blowing-assisted electro-spinning and non-toxic post treatments. Polymer 2005; 46: 4853-67.

[13] Xu S, Li J, He A, Liu W, Jiang X, Zheng J, Han CC, Hsiao BS, Chu B, Fang D. Chemical crosslinking and biophysical properties of electrospun hyaluronic acid based ultra-thin fibrous membranes. Polymer 2009; 50: 3762-3769.

[14] Ji Y, Ghosh K, Li B, Sokolov, JC, Clark, RA, Rafailovich MH. Dual-syringe reactive electrospinning of cross-linked Hyaluronic Acid hydrogel nanofibers for tissue engineering applications. Macromol Biosci 2006; 6: 811-7.

[15] Colins MN, Birkinshaw C. Comparison of the effectiveness of four different crosslinking agents with hyaluronic acid hydrogel films for tissue-culture applications. J Appl Polym Sci. 2007; 104: 3183-91.

[16] Arnal-Pastor M, Vallés-Lluch A., Keicher M, Monleón Pradas M. Coating typologies and constrained swelling of hyaluronic acid gels within scaffold pores. J. Colloid and Interf Sci 2011; 361: 361-69.

[17] Schofer MD, Roessler PP, Schaefer J, Theisen C, Schlimme S, Heverhagen JT, Voelker M, Dersch R, Agarwal S, Fuchs-Winkelmann S, Paletta JRJ. Electrospun PLLA Nanofiber scaffolds and their use in combination with BMP-2 for reconstruction of bone defects. PLoS ONE 2011; 6 : e25462.

[18] Cheung HY, Lau KT, Lu TP, Hui D. A critical review on polymer-based bio-engineered materials for scaffold development. Compos Part B-Eng 2007; 38: 291-300. 
[19] Khademhosseini A, Eng G, Yeh J, Kucharczyk PA, Langer R, Vunjak-Novakovic G, Radisic M. Microfluidic patterning for fabrication of contractile cardiac organoids. Biomed Microdevices 2007; 9: 149-57.

[20] Reijnen MM, Bleichrodt RP, van Goor H. Pathophysiology of intra-abdominal adhesion and abscess formation, and the effect of hyaluronan. Br J Surg. 2003 May;90(5):533-41.

[21] Greenspan L. Humidity fixed points of binary saturated aqueous solutions. J. Res. Nat. Bur. Stand A Phys. Chem. 1977; 81A: 89-95.

[22] Bölgen N, Vargel I, Korkusuz P, Menceloğlu YZ, Pişkin E. In Vivo Performance of Antibiotic Embedded Electrospun PCL Membranes for Prevention of Abdominal Adhesions. J Biomed Mater Res Part B: Appl Biomater. 2007; 81B: 530-543

[23] Munir MM, Iskandar F, Yun KM, Okuyama K, Abdullah M. Optical and electrical properties of indium tin oxide nanofibers prepared by electrospinning. Nanotechnology 2008; 19(14): 145603 . doi: 10.1088/0957-4484/19/14/145603.

[24] Diego RB, Estellés JM, Sanz JA, García-Aznar JM, Sánchez MS. Polymer scaffolds with interconnected spherical pores and controlled architecture for tissue engineering: fabrication, mechanical properties, and finite element modeling. J Biomed Mater Res B Appl Biomater 2007; 81: 448-55.

[25] Gong Y., Zhou Q., Gao C., Shen J. In vitro and in vivo degradability and cytocompatibility of poly(l-lactic acid) scaffold fabricated by a gelatin particle leaching method. Acta Biomaterialia 2007; 3(4), 531-540.

[26] Diamond M P., Burns E.L., Accomando B., Mian S., Holmdahl L. Seprafilm® adhesion barrier: (1) a review of preclinical, animal, and human investigational studies. Gynecol Surg 2012; 9: 237-245. doi: 10.1007/s10397-012-0741-9 
[27] Caspi O, Lesman A, Basevitch Y, Gepstein A, Arbel G, Habib IH, Gepstein L, Levenberg S.

Tissue engineering of vascularized cardiac muscle from human embryonic stem cells. Circ Res. 2007; 100(2): 263-72. 